\title{
Diagnosis of early gastric cancer using image enhanced endoscopy: a systematic approach
}

\author{
Masaki Miyaoka ${ }^{1}$, Kenshi Yao ${ }^{1}$, Hiroshi Tanabe ${ }^{2}$, Takao Kanemitsu ${ }^{1}$, Kensei Otsu ${ }^{3}$, Kentaro Imamura ${ }^{3}$, \\ Yoichiro Ono $^{3}$, Satoshi Ishikawa ${ }^{3}$, Tatsuhisa Yasaka ${ }^{3}$, Toshiharu Ueki ${ }^{3}$, Atsuko Ota ${ }^{2}$, Seiji Haraoka $^{2}$, \\ Akinori Iwashita ${ }^{2}$ \\ ${ }^{1}$ Department of Endoscopy, ${ }^{2}$ Department of Pathology, ${ }^{3}$ Department of Gastroenterology, Fukuoka University Chikushi Hospital, Chikushino City, \\ Fukouka, Japan \\ Contributions: (I) Conception and design: K Yao; (II) Administrative support: M Miyaoka, K Yao; (III) Provision of study materials or patients: \\ M Miyaoka, K Yao, T Ueki; (IV) Collection and assembly of data: M Miyaoka, K Yao; (V) Data analysis and interpretation: M Miyaoka, K Yao; \\ (VI) Manuscript writing: All authors; (VII) Final approval of manuscript: All authors. \\ Correspondence to: Kenshi Yao, MD, PhD. Department of Endoscopy, Fukuoka University Chikushi Hospital, 1-1-1 Zokumyoin, Chikushino City, \\ Fukuoka 818-8502, Japan. Email: yao@fukuoka-u.ac.jp.
}

\begin{abstract}
This paper provides an overview of the principles of a vessel plus surface (VS) classification system to explain the diagnostic system of early gastric cancer using image-enhanced magnifying endoscopy. Furthermore, this paper introduces the magnifying endoscopy simple diagnostic algorithm for gastric cancer (MEADA-G) developed according to the VS classification system, with a description of the procedures performed for diagnosis. In addition to the diagnostic system, white opaque substance (WOS), light blue crest (LBC), white globe appearance (WGA), and vessels within epithelial circle (VEC) patterns, which are representative findings that can be observed in the gastric mucosa by image-enhanced magnifying endoscopy, are also described. Image-enhanced magnifying endoscopy is particularly useful in the diagnosis of differentiated-type early gastric cancer. It is important to use the appropriate clinical strategies based on a comprehensive understanding of the usefulness and limitations of the diagnostic system described in this paper.
\end{abstract}

Keywords: Image-enhanced endoscopy; magnifying endoscopy; early gastric cancer; diagnostic system

Received: 24 September 2019; Accepted: 13 November 2019; Published: 05 October 2020.

doi: $10.21037 / \operatorname{tgh} .2019 .12 .16$

View this article at: http://dx.doi.org/10.21037/tgh.2019.12.16

\section{Introduction}

Endoscopic diagnosis of early gastric cancer mainly comprises two steps: (I) detection of cancer and (II) differentiation between cancerous and non-cancerous lesions. The usefulness of image-enhanced endoscopic observation in screening endoscopy to detect early gastric cancer remains unclear, but a large-scale clinical study on this issue is currently underway. On the other hand, the usefulness of image-enhanced magnifying endoscopy in differential diagnosis of gastric cancer has already been established (1-13). Therefore, this paper provides an overview of the diagnostic system for early gastric cancer and presents the characteristic findings of gastric mucosal lesions visualized by image-enhanced magnifying endoscopy, with a focus on differentiation between cancerous and non-cancerous lesions using image-enhanced magnifying endoscopy.

\section{Diagnostic system}

\section{VS classification system}

The vessel plus surface (VS) classification system is an established diagnostic system for differentiation between cancerous and non-cancerous lesions using magnifying endoscopy; this system was proposed by Yao et al. in 2009 (1). The usefulness of this system for diagnosing early 
A

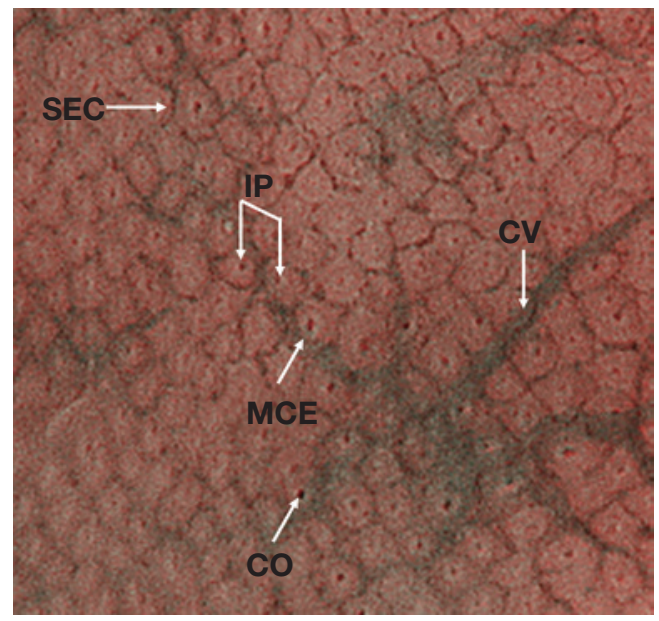

C

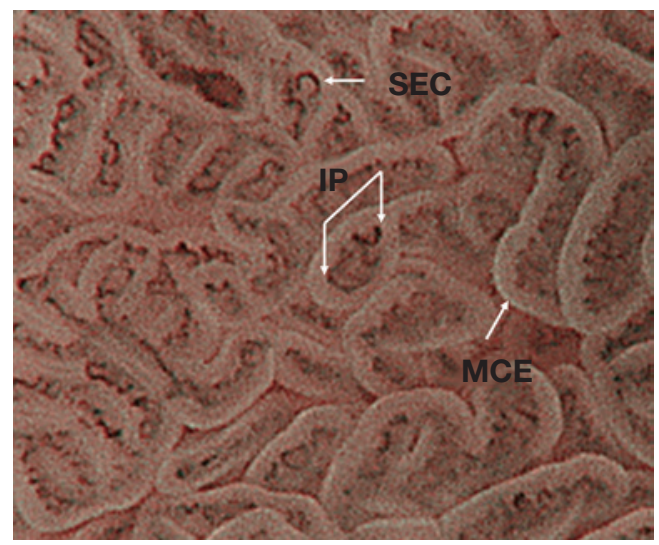

B

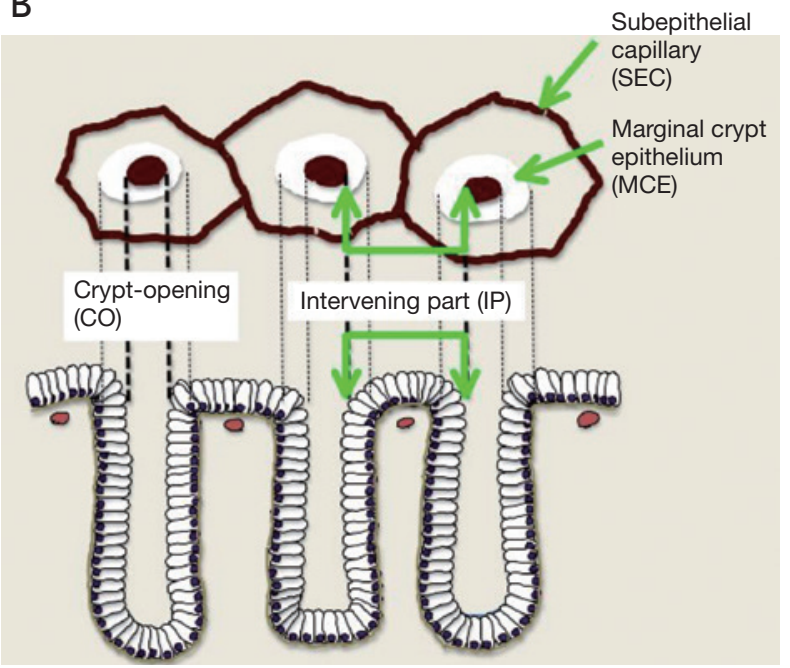

D

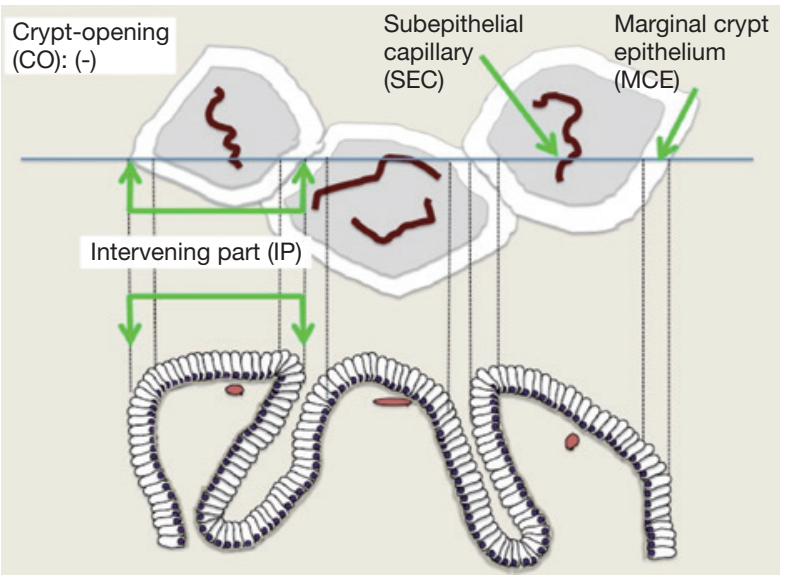

Figure 1 Microanatomies as visualized using magnifying endoscopy with narrow band imaging (NBI) in the stomach. (A) Magnifying endoscopic image with NBI of normal gastric fundic gland mucosa. (B) Correspondence between the magnifying endoscopic image with NBI and histological findings of normal gastric fundic gland mucosa. (C) Magnifying endoscopic image with NBI of normal gastric pyloric gland mucosa. (D) Correspondence between the NBI-combined magnifying endoscopic image and histological findings of normal gastric pyloric gland mucosa. NBI, narrow-band imaging; SEC, subepithelial capillary; MCE, marginal crypt epithelium; CO, crypt opening; CV, collecting venule; IP, intervening part. [Reprinted from (14)].

gastric cancer has been verified with high-level evidence $(4,5)$. The most useful features of this diagnostic system are as follows: (I) it enables the diagnosis of minute cancers $\leq 5 \mathrm{~mm}$ in size and of early gastric cancers of the superficial flat (0-IIb) type among gastritis-like cancers (superficial type of early gastric cancer, 0-II types), which cannot be diagnosed using conventional endoscopy; (II) it enables preoperatively necessary determination of the borders of early gastric cancer.

The fundamental principle of the VS classification system is to evaluate the microvascular (MV) and microsurface (MS) patterns, using anatomical terms for the analysis of findings on magnifying endoscopy of the stomach (Figure 1). The terms used for the analysis of the MV pattern include the following: (I) subepithelial capillary network (SEC), (II) collecting venule (CV), and (III) microvessels (MVs). The terms used for the analysis of the MS pattern include the following: (I) marginal crypt epithelium (MCE), (II) crypt opening (CO), (III) intervening part between crypts (IP), (IV) light blue crest (LBC), and (V) white opaque 


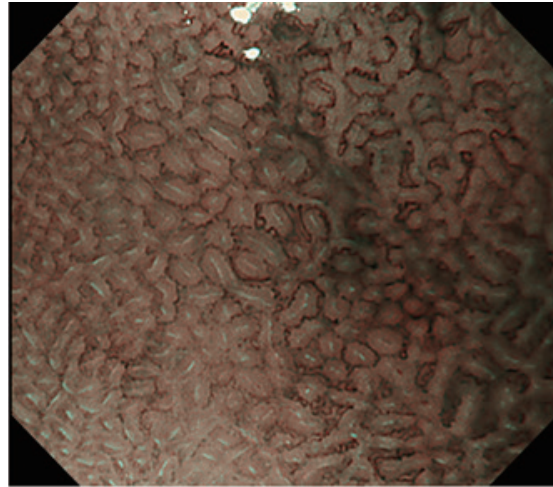

Regular

S

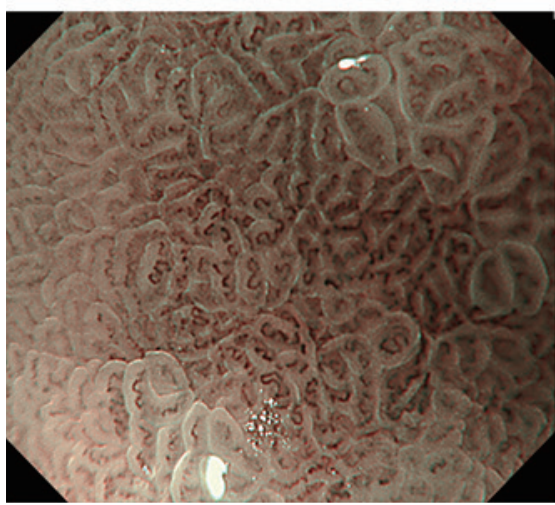

Regular

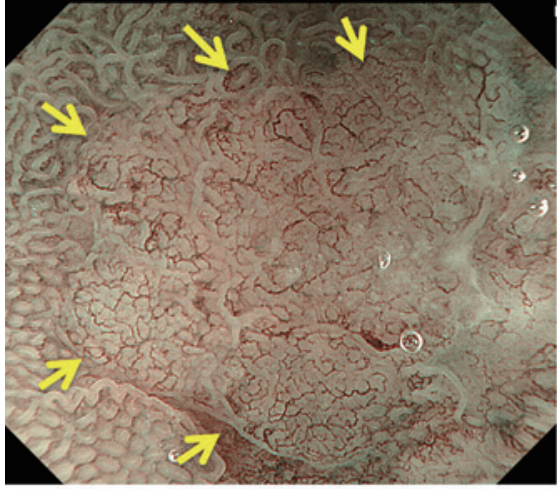

Irregular

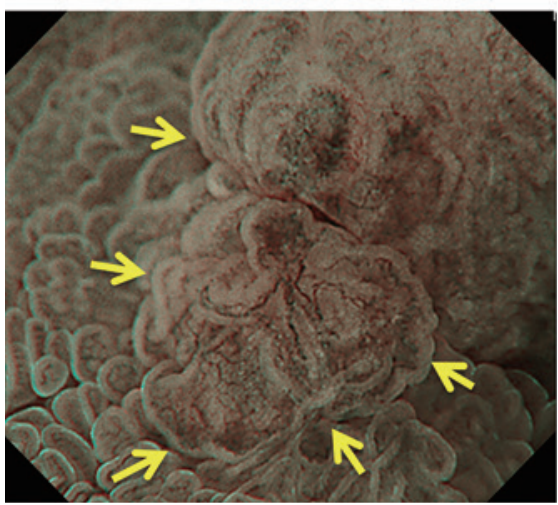

Irregular

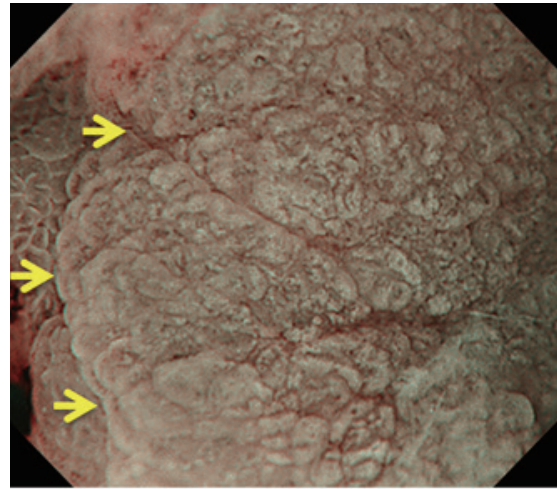

Absent

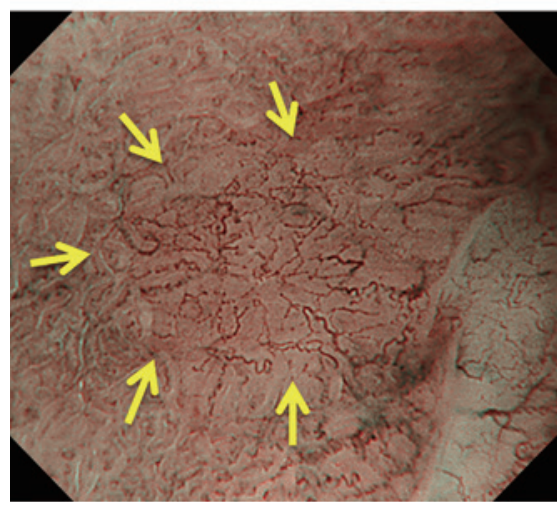

Absent

Figure 2 VS classification using magnifying endoscopy with NBI. The microvascular pattern (V) is classified as regular/irregular/absent, as is the microsurface pattern (S) (the yellow arrows denote a demarcation line). VS, vessel plus surface; NBI, narrow-band imaging. [Reprinted from (3)].

substance (WOS).

According to the VS classification system, the characteristic endoscopic findings of early gastric cancer include the presence of a clear demarcation line between cancerous and non-cancerous mucosae and the presence of an irregular MV pattern and/or irregular MS pattern inside the demarcation line. The demarcation line is defined as a borderline recognizable by the abrupt change in the $\mathrm{MV}$ or MS pattern between the lesion and non-lesion areas. The MV and MS patterns are evaluated separately regarding whether they are regular, irregular, or absent, and lesions that meet the following diagnostic criteria are diagnosed as cancerous, whereas those that do not are diagnosed as noncancerous.

\section{Diagnostic criteria}

(I) Presence of an irregular MV pattern with a demarcation line;
(II) Presence of an irregular MS pattern with a demarcation line.

Lesions that meet criterion (I) or (II) (or both) are diagnosed as cancerous lesions, whereas others are diagnosed as non-cancerous lesions.

It has been reported that $97 \%$ of early gastric cancers theoretically meet these diagnostic criteria (1).

As shown previously, the MV and MS patterns can be classified into three categories: regular/irregular/absent (Figure 2). In the regular MV pattern, the capillaries under each epithelium have a closed loop (polygonal) or open loop morphology, showing a regular arrangement of symmetrically distributed homogeneous shapes. Meanwhile, the irregular MV pattern is characterized by a closed loop (polygonal), open loop, tortuous, branching, or irregular morphology. MVs have heterogeneous shapes, and they are distributed asymmetrically, showing an irregular arrangement. Cases in which there are no observable 


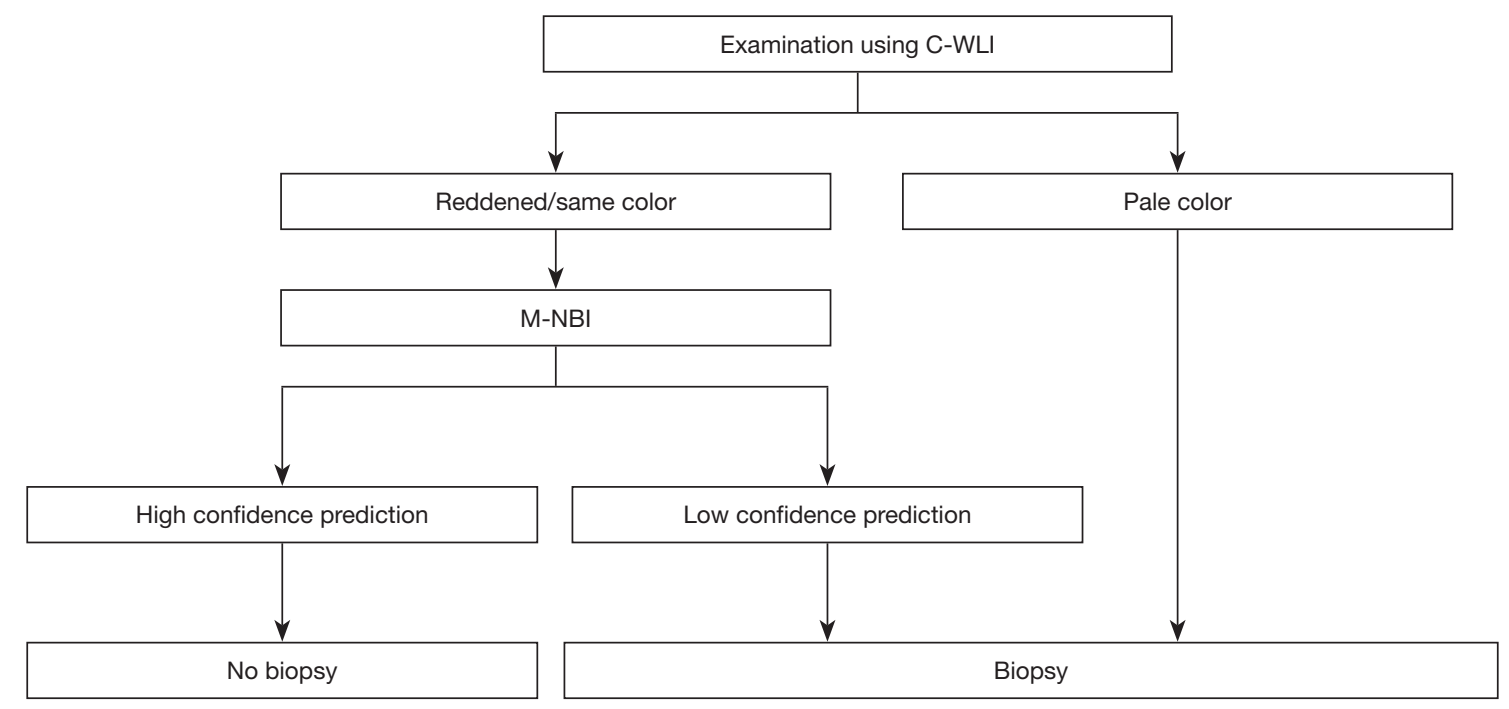

Figure 3 A strategy for magnifying endoscopy with NBI in screening gastroscopy. C-WLI, conventional endoscopy with white light imaging; M-NBI, magnifying endoscopy with narrow-band imaging; NBI, narrow-band imaging. [Reprinted from (5)].

subepithelial MVs are judged as absent MV pattern. In such cases, the MS pattern (showing features such as MCE, WOS, and LBC), instead of the MV pattern, is used as the indicator for magnifying endoscopic diagnosis (2).

Although MCE is often used for the judgment of the MS pattern, other findings such as CO, IP, LBC, and WOS can also be used if they are present. In the regular MS pattern, the morphology of each MCE is curved or oval, showing homogeneous shapes, symmetrical distribution, and regular arrangement. In the irregular MS pattern, the morphology of each MCE is curved or oval, or villous on rare occasions, showing heterogeneous shapes, an asymmetrical distribution, and an irregular arrangement. When no MS pattern including MCE is observable, the case is judged as absent MS pattern. In such cases, the MV pattern is necessarily well visualized; therefore, the features of the MV pattern, instead of the MS pattern, are used as indicators for magnifying endoscopic diagnosis (1).

It has also been shown in a multicenter prospective study that some lesions are difficult to observe by magnifying endoscopy using the VS classification system (5). Such lesions are discolored, flat undifferentiated-type cancer lesions. Taking into account clinical limitations, we have proposed a clinical strategy as shown in Figure 3.

Specifically, we have suggested that biopsy is necessary for discolored, flat mucosal lesions, whereas magnifying endoscopy of the stomach can be used as an optical biopsy in cases of other lesions as long as there is high-confidence prediction. The magnifying endoscopy simple diagnostic algorithm for gastric cancer (MESDA-G) described below should also be applied according to this clinical strategy. Figure 4 shows discolored, flat undifferentiated-type gastric cancer, which is difficult to diagnose by magnifying endoscopy using the VS classification system.

\section{MESDA-G using the VS classification system}

Although numerous diagnostic systems aimed at magnifying endoscopic diagnosis of early gastric cancer have been proposed in Japan and elsewhere, no consensus had been reached on an established and standardized set of diagnostic criteria or diagnostic system. Against this background, the Japanese Gastroenterological Association Guidelines Subcommittee performed a systematic review and selected the terms and diagnostic system to be used for magnifying endoscopic diagnosis of the stomach in evidence-based medicine. Consequently, the VS classification system was adopted as a diagnostic system, together with the anatomical terms used for this system. On this basis, MESDA-G, was proposed (3). This algorithm was endorsed by the Japanese Gastroenterological Association, Japan Gastroenterological Endoscopy Society, Japanese Gastric Cancer Association, and World Endoscopy Organization, and a consensus was reached on its status as a standardized algorithm. Figure 5 shows the algorithm. First, white-light magnifying endoscopy is used to observe the inside of the 

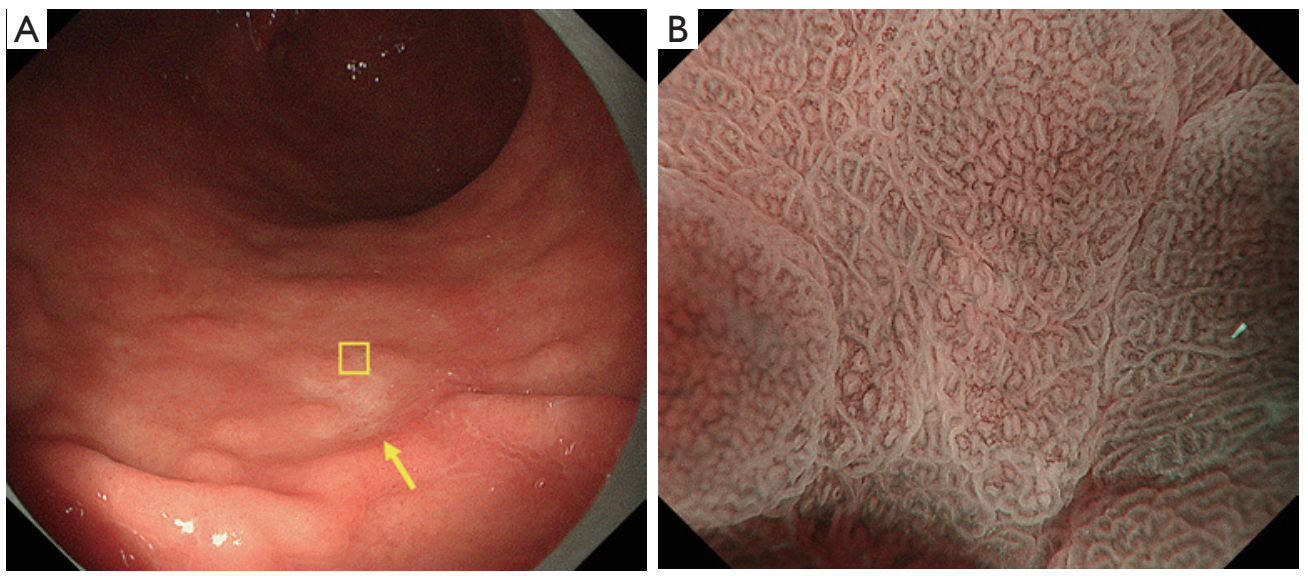

Figure 4 Difficult case of magnifying endoscopic diagnosis using the VS classification system. Demarcation line, absent, irregular MV pattern, absent, irregular MS pattern, absent. (A) Conventional endoscopic image. A well-demarcated slightly depressed pale lesion is present in the greater curvature of the gastric antrum (yellow arrow). (B) Magnifying endoscopic image with NBI [the highest magnification of the yellow squared area of (A)]. It was judged that there are no abrupt changes in capillaries underneath the mucosal epithelium or MCE in both background mucosae to the lesion. Accordingly, a demarcation line is absent. The shapes of individual microvessels are homogeneous, showing a symmetrical distribution and regular arrangement. Thus, the pattern was judged to be a regular MV pattern. Regarding the MS pattern, the marginal crypt epithelium is oval, showing homogeneous shapes, a symmetrical distribution, and a regular arrangement. Thus, the pattern was judged to be a regular MS pattern. Therefore, the lesion had the regular MV pattern plus regular MS pattern without a demarcation line, and according to the VS classification system, a diagnosis of non-cancerous lesion was made. However, histopathologically, the lesion was diagnosed as signet ring cell carcinoma. These discolored undifferentiated-type cancer lesions represent difficult cases to diagnose using magnifying endoscopy, requiring a biopsy to establish a definitive diagnosis. VS, vessel plus surface; MV, microvascular; MS, microsurface; NBI, narrow-band imaging; MCE, marginal crypt epithelium.

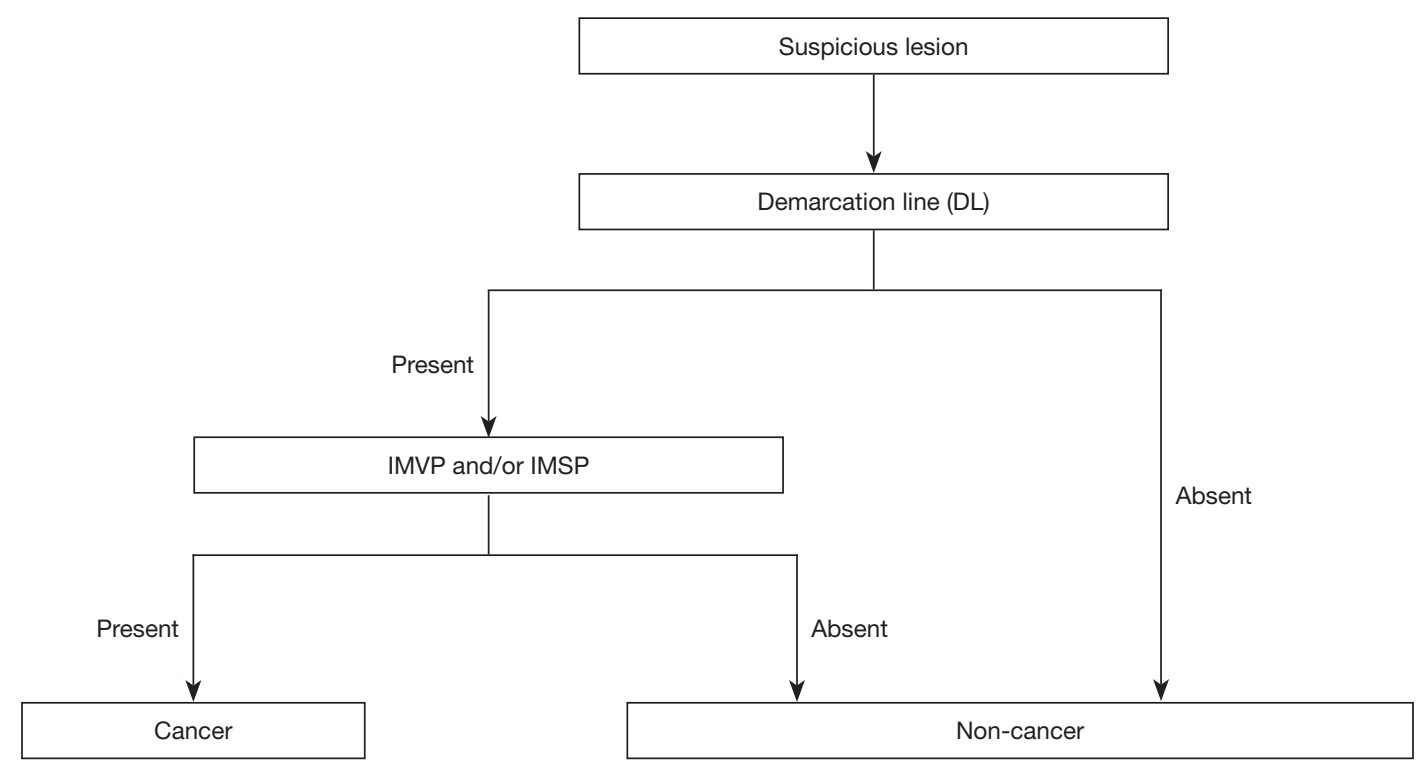

Figure 5 Magnifying endoscopy simple diagnostic algorithm for early gastric cancer (MESDA-G). VS, vessel plus surface; IMVP, irregular microvascular pattern; IMSP, irregular microsurface pattern. [Reprinted with some modifications from (3)]. 

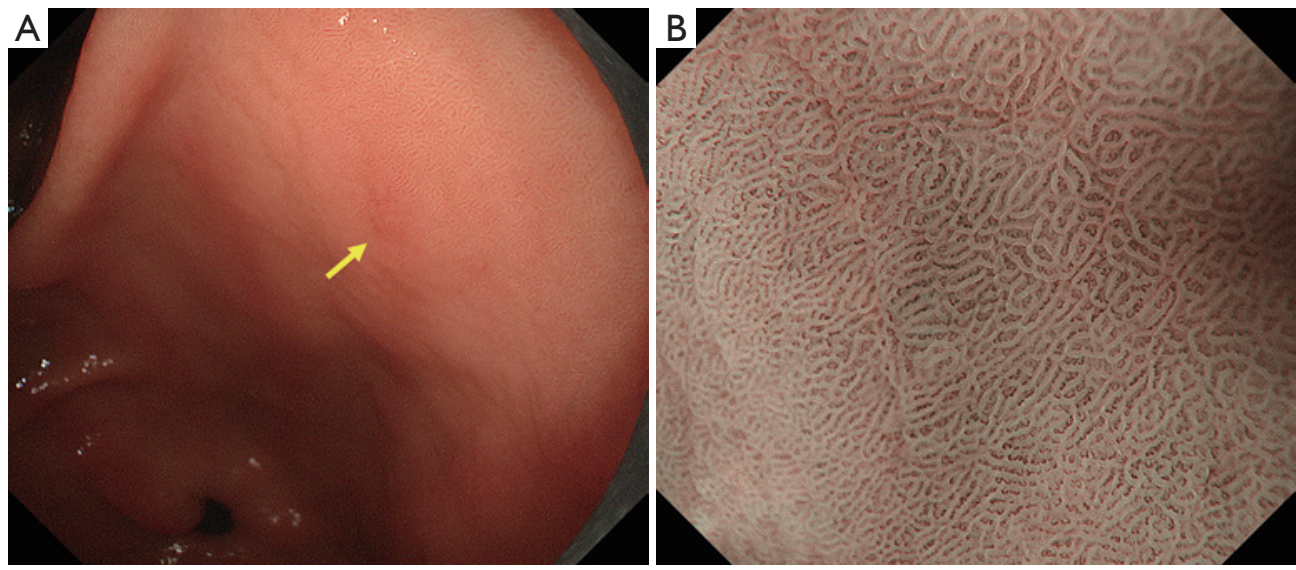

Figure 6 Example of application of MESDA-G (I). Demarcation line: absent. (A) Conventional endoscopic image. A flat reddish mucosal lesion is present in the posterior wall of the gastric antrum (yellow arrow). (B) Magnifying endoscopic image with NBI. Subepithelial capillaries are gradually dilated in non-reddish to reddish areas. Furthermore, there is a gradual change in the shape of MCE. More specifically, there is no identifiable abrupt change in the border area between the MV and MS patterns. Thus, the demarcation line was judged to be absent. A diagnosis of non-cancerous lesion was made according to MESDA-G. MESDA-G, magnifying endoscopy simple diagnostic algorithm for gastric cancer; NBI, narrow-band imaging; MCE, marginal crypt epithelium; MV, microvascular; MS, microsurface.

stomach according to the systematic screening protocol for the stomach (15) to perform examinations for any lesions suggestive of early gastric cancer. If such a suspicious lesion is detected, magnifying endoscopy should be performed to differentiate between cancerous and non-cancerous lesions. More specifically, the presence/absence of a demarcation line should be determined using magnifying endoscopy (Figure 5). If no demarcation line is identified, the lesion should be diagnosed as a non-cancerous one. If there is a demarcation line, the MV and MS patterns inside the demarcation line should be separately examined regarding whether they are regular, irregular, or absent. If the irregular MV pattern and/or irregular MS pattern is identified, the lesion is diagnosed as cancer, whereas if these patterns are not identified, the lesion is diagnosed as a noncancerous one. This is a truly simple and clear algorithm. Figures 6-8 provide actual endoscopic findings to show the procedures performed for diagnosis based on MESDA-G.

\section{Characteristic findings of the gastric mucosa on image-enhanced magnifying endoscopy}

\section{WOS}

WOS is a white substance that obscured subepithelial MVs present in the superficial layer of the mucosa clearly visualized on magnifying endoscopy with narrow-band imaging (NBI). The presence of WOS in the gastric mucosa was first reported by Yao et al. in 2008. This finding is often obtained in intestinal metaplasia in the mucosa of chronic gastritis and in gastric epithelial tumors (adenoma or cancer) with intestinal phenotype $(2,16,17)$. The nature of WOS is a phenomenon recognized as white substance observed through the strong scattering or reflection of the projected light from the endoscopy on minute fat droplets accumulated in and under the epithelium $(18,19)$. When WOS is present, the projected light cannot reach subepithelial MVs, resulting in poor visibility of the vessels. Differences in the morphological features of WOS serve as useful indicators in the differential diagnosis of cancer and adenoma. More specifically, cancerous lesions have irregular WOS, whereas low-grade adenomatous lesions have regular WOS (2). In addition, it has been reported that WOS-positive gastric epithelial tumors have a mucous character of some intestinal phenotype (intestinal type or gastrointestinal phenotype) (18) and that WOS serves as an indicator of differentiated-type cancers because there is no WOS in undifferentiated-type cancers (20). Figures 9 and 10 show actual endoscopic findings.

\section{$L B C$}

It has been shown that magnifying endoscopy with NBI is 


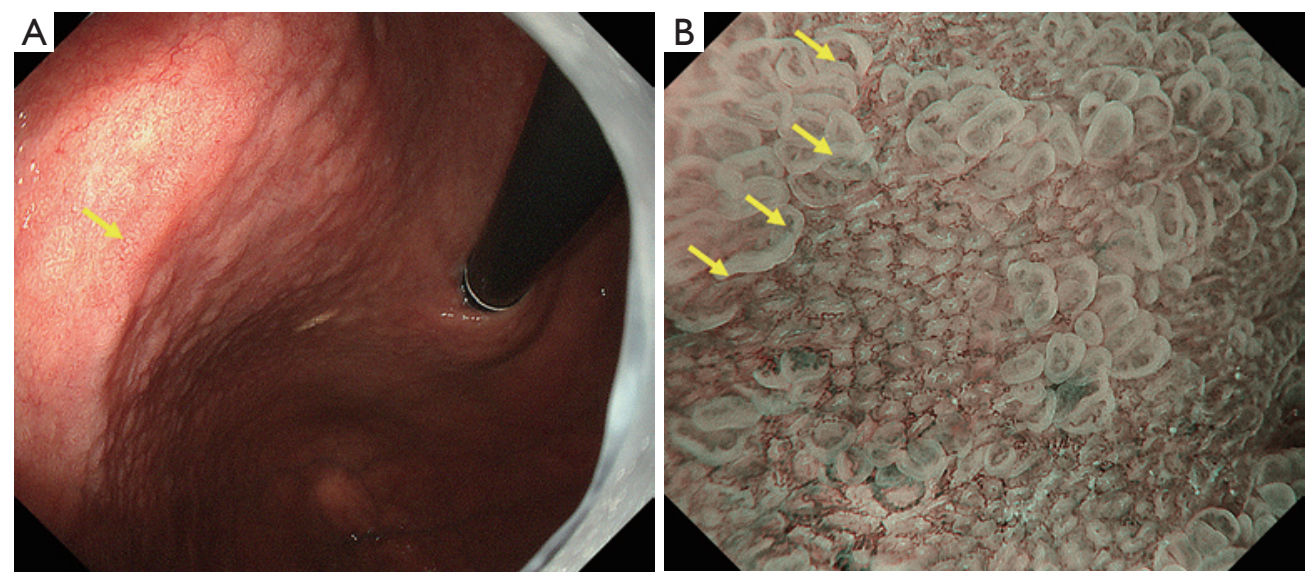

Figure 7 Example of application of MESDA-G (II). Demarcation line: present; irregular MV pattern: absent; irregular MS pattern: absent. (A) Conventional endoscopic image. A regional, slightly concave reddish lesion is present in the posterior wall of the gastric corpus (yellow arrow). (B) Magnifying endoscopic image with NBI. There is an abrupt change in the MV and MS patterns at the site indicated by the yellow arrows. Namely, a demarcation line is present. In this case, the VS classification of the MV and MS patterns inside the demarcation line was carried out according to MESDA-G to judge the presence or absence of the irregular MV pattern and/or irregular MS pattern. In the MV pattern, the shapes of individual microvessels are homogeneous, showing a symmetrical distribution and regular arrangement. Therefore, this case was judged to have a regular MV pattern. Regarding the MS pattern, the MCE has an oval morphology, showing homogeneous shapes, a symmetrical distribution, and a regular arrangement. There are regular light blue crests in glandular openings arranged regularly. Based on these findings, the case was judged to have a regular MS pattern. Therefore, the lesion was judged to have a regular MV pattern plus regular MS pattern according to the VS classification system, resulting in a diagnosis of non-cancerous lesion. MESDA-G, magnifying endoscopy simple diagnostic algorithm for gastric cancer; MV, microvascular; MS, microsurface; NBI, narrow-band imaging; VS, vessel plus surface; MCE, marginal crypt epithelium.

useful for the diagnosis of intestinal metaplasia (21-23). A comparison of magnifying endoscopy with NBI and whitelight magnifying endoscopy has revealed the superiority of the former over the latter for correctly diagnosis of intestinal metaplasia (24). The LBC sign was first reported by Uedo et al. in 2006 (25). According to their report, LBC is defined as blue-white lines of light observed at the edge of MCE on magnifying endoscopy with NBI (Figure 11). The LBC sign is considered to be a phenomenon that occurs when the brush border of the absorptive epithelial surface of histopathologically CD10-positive intestinal metaplasia reflects narrow-band light having a central wavelength of $415 \mathrm{~nm}$. Regarding the diagnostic ability of LBC for histopathological intestinal metaplasia, the reported sensitivity and specificity are $89 \%$ and $93 \%$, respectively (25). In addition, Kanemitsu et al. used a combination of LBC and WOS for the diagnosis of intestinal metaplasia, and reported a sensitivity and specificity of $87.5 \%$ and $93.8 \%$, respectively (17). Moreover, meta-analyses including studies from outside of Japan also confirmed the excellent diagnostic ability of LBC in the diagnosis of intestinal metaplasia (26-28). The LBC sign is also useful for determining the tumor border; the borderline devoid of LBC often matches the tumor borderline (Figure 12).

\section{White globe appearance (WGA)}

WGA, if present inside the demarcation line, is a highly specific finding on magnifying endoscopy that allows differential diagnosis between differentiated-type cancerous and non-cancerous lesions. WGA is defined as a small (measuring $\leq 1 \mathrm{~mm}$ ) white globular appearance just beneath the epithelium found on magnifying endoscopy with NBI. WGA was first reported by Doyama et al. in 2015 (29). It corresponds to so-called intraglandular necrotic debris, which is a markedly dilated tumor gland duct containing retained eosinophilic necrotic material observed on close histopathological examination. WGA is a highly specific cancer marker for magnifying endoscopy. In other words, the presence of WGA allows differential diagnosis between 

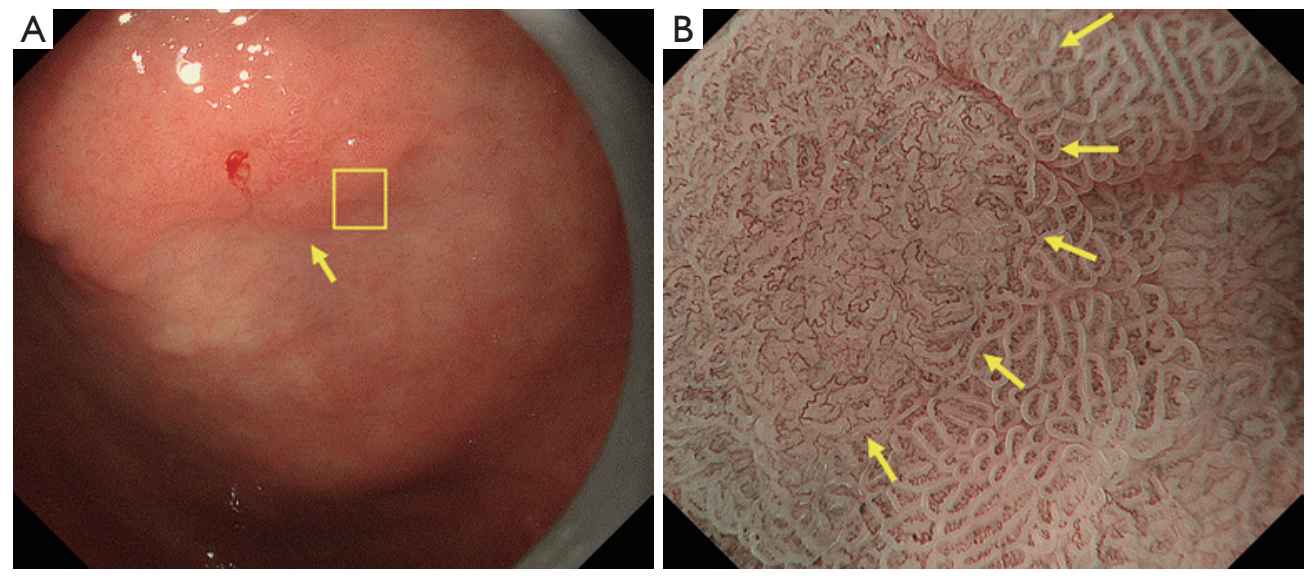

Figure 8 Example of application of MESDA-G (III). Demarcation line: present; irregular MV pattern: present; irregular MS pattern: present. (A) Conventional endoscopic image. There is a well-demarcated depressed lesion accompanied by irregular redness in the lesser curvature of the gastric antrum (yellow arrow). (B) Magnifying endoscopic image with NBI [yellow square area in (A)]. A clear demarcation line is seen in the border area (yellow arrows). Regarding the MV pattern inside the demarcation line, each microvessel has an irregular loop morphology. The microvessels show heterogeneous shapes, an asymmetrical distribution, and an irregular arrangement. Therefore, the pattern was judged to be an irregular MV pattern. Regarding the MS pattern, there are some areas devoid of MCE, which were judged to have an absent MS pattern. In areas where MCE is visible, each has an arcuate MCE morphology, that is, shapes of MCE are heterogeneous, with an asymmetrical distribution and irregular arrangement. The case was judged to have an absent/irregular MS pattern. Based on these findings, the pattern was judged to be an irregular MV pattern plus absent/irregular MS pattern according to the VS classification system, and a diagnosis of cancer was made. MESDA-G, magnifying endoscopy simple diagnostic algorithm for gastric cancer; MV, microvascular; MS, microsurface; NBI, narrow-band imaging; MCE, marginal crypt epithelium; VS, vessel plus surface.
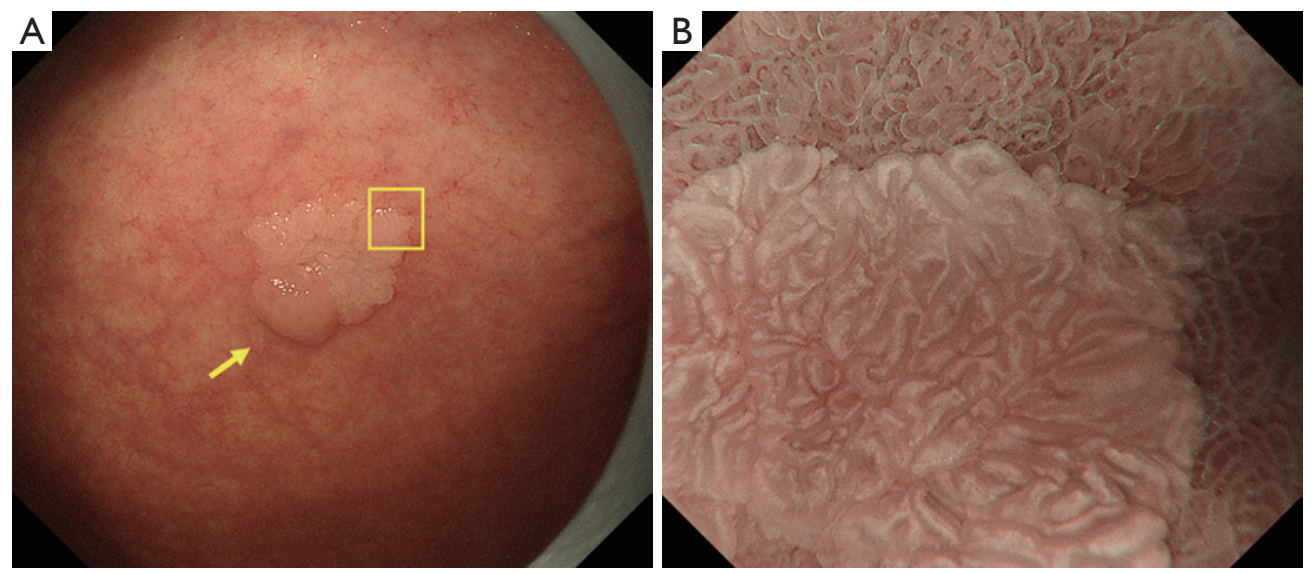

Figure 9 Low-grade adenoma having regular WOS (non-cancer). (A) Conventional endoscopic image (white light). A discolored, flatelevated lesion (yellow arrow) is present in the anterior wall in the lower part of the gastric corpus. (B) Magnifying endoscopic image with NBI [the highest magnification of the yellow squared area of (A)]. WOS inside the demarcation line is highly dense and large and is localized in the intervening part. WOS shows homogeneous shapes, a symmetrical distribution, and a regular arrangement, leading to the judgment of a regular MS pattern (regular WOS). Microvascular structures are not visible because of the presence of WOS; therefore, the pattern was judged to be an absent MV pattern. According to the VS classification system, the result was an absent MV pattern plus regular MS pattern with a demarcation line, and a diagnosis of non-cancerous lesion was made. Histopathologically, the lesion was a low-grade tubular adenoma. WOS, white opaque substance; NBI, narrow-band imaging; MS, microsurface; MV, microvascular; VS, vessel plus surface. 


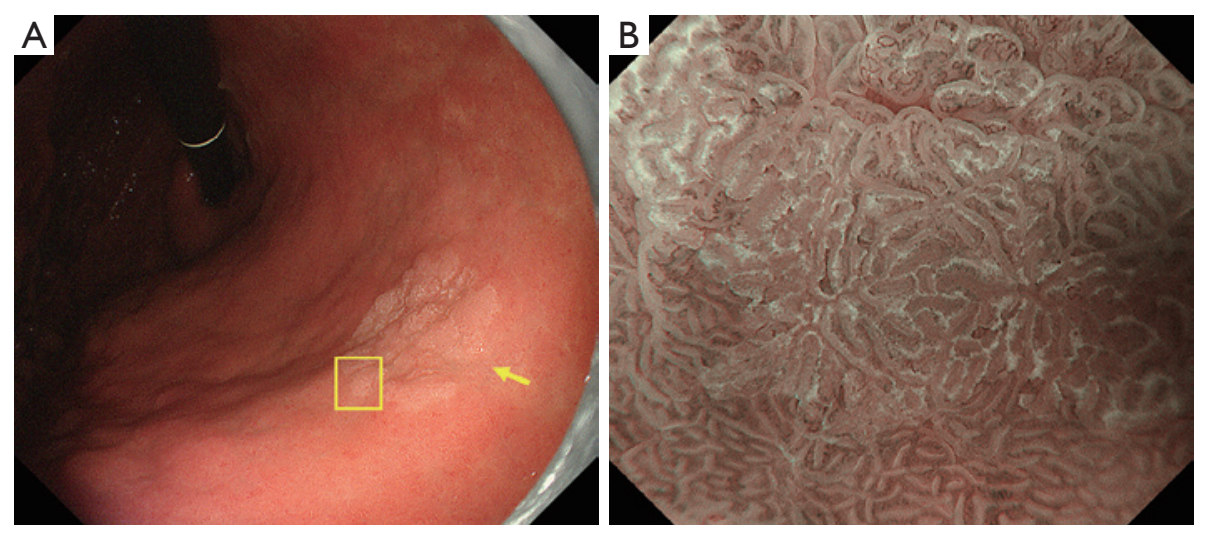

Figure 10 Well-differentiated adenocarcinoma (cancer) judged to have irregular WOS. (A) Conventional endoscopic image (white light). A discolored, elevated lesion (yellow arrow) is present in the lesser curvature in the lower part of the gastric corpus. (B) Magnifying endoscopic image with NBI [the highest magnification of the yellow squared area shown in (A)]. WOS inside the demarcation line shows heterogeneous shapes, a symmetrical distribution, and an irregular arrangement, leading to the judgment of an irregular MS pattern (irregular WOS). Microvascular structures are not visible because of the presence of WOS, and the pattern was judged to be an absent MV pattern. According to the VS classification system, the result was an absent MV pattern plus irregular MS pattern with a demarcation line, leading to a diagnosis of cancer. Histopathologically, the lesion was very-well-differentiated tubular adenocarcinoma. WOS, white opaque substance; NBI, narrowband imaging; MS, microsurface; MV, microvascular; VS, vessel plus surface.
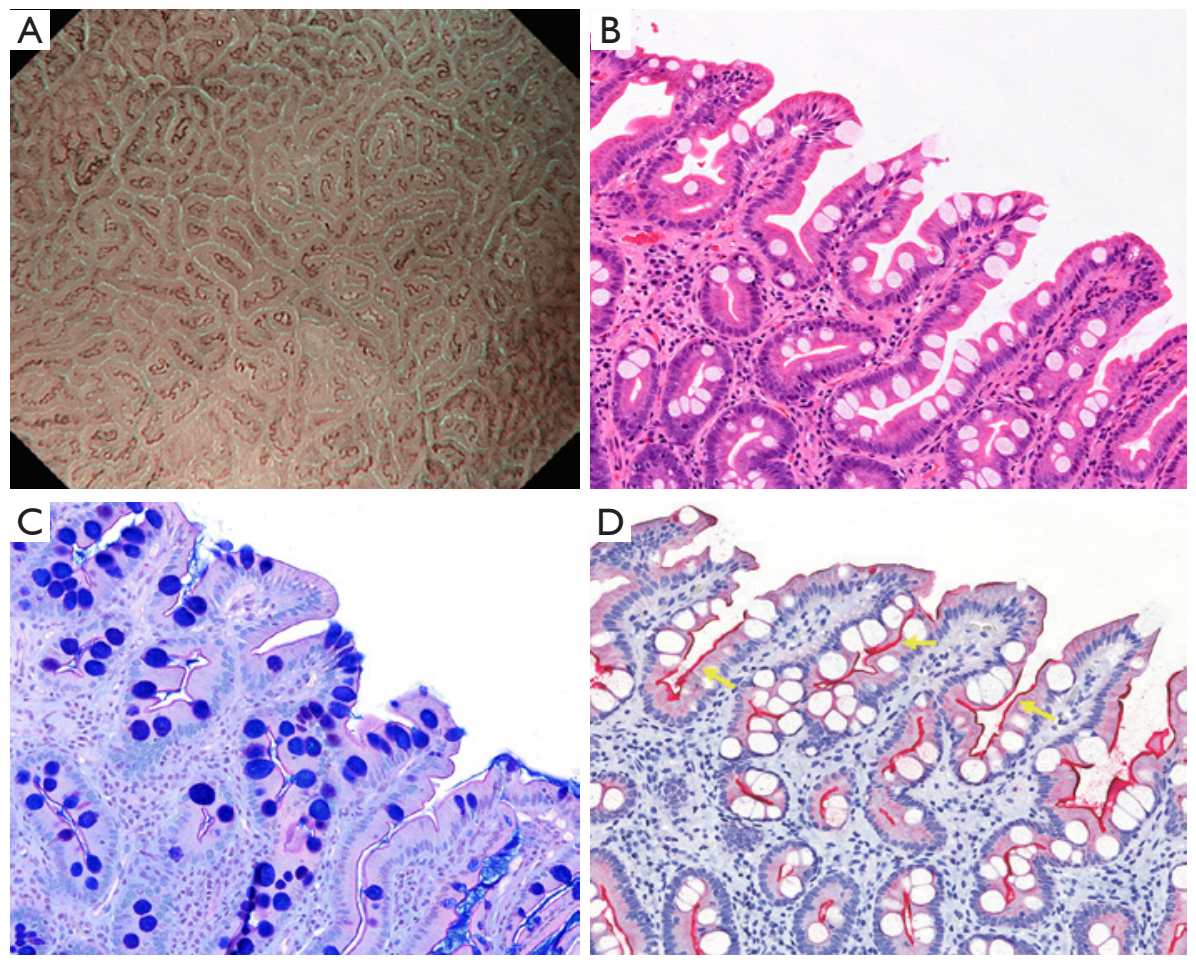

Figure 11 Mucosa that is positive for LBC. (A) Magnifying endoscopic image with NBI showing LBC at the edge of the MCE. (B) Histopathological appearance with hematoxylin and eosin $(\mathrm{H} \& \mathrm{E})$ staining (magnification $\times 20)$ showing goblet cells within the crypt epithelium, and a brush border visible at the epithelial surface. (C) Histopathological appearance with Alcian blue periodic acidSchiff staining (magnification $\times 20$ ) showing goblet cells and a clearly visible brush border. (D) Immunohistochemical staining (CD10, magnification $\times 20$ ): the brush border (yellow arrows) on the absorption epithelium surface of intestinal metaplasia becomes CD10 positive. LBC, light blue crest; NBI, narrow-band imaging; MCE, marginal crypt epithelium. [Reprinted from (17)]. 

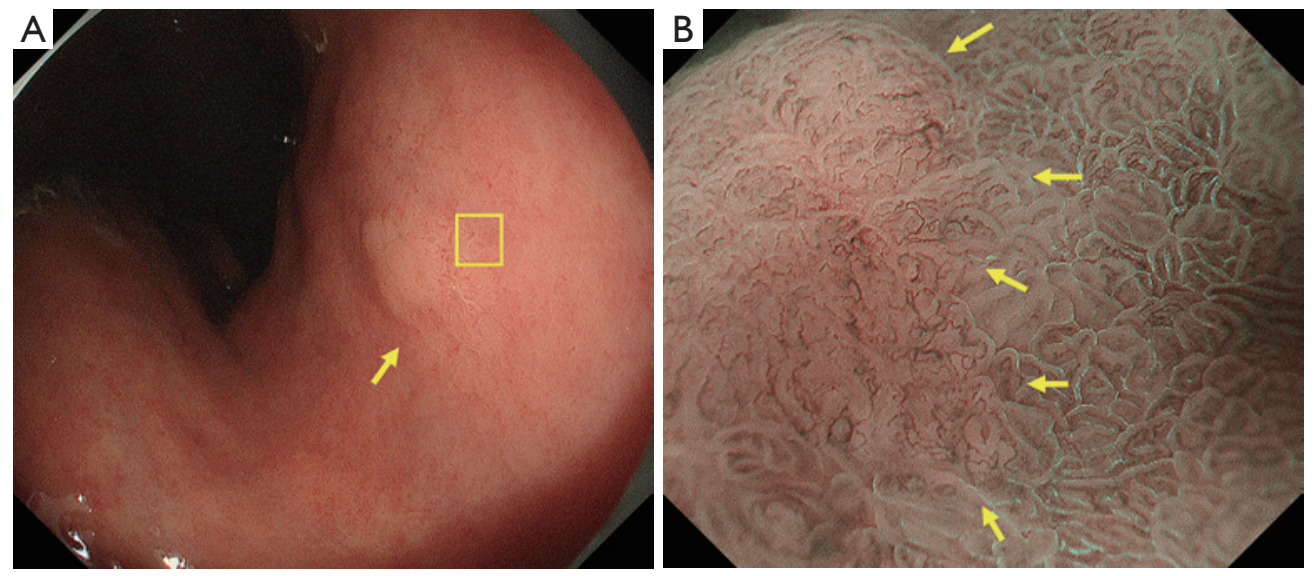

Figure 12 Low-grade adenoma(non-cancer) in which LBC is useful for border of the tumor. (A) Conventional endoscopic image (white light). A discolored, flat-elevated lesion (yellow arrow) is present in the lesser curvature in the gastric corpus. (B) Magnifying endoscopic image with NBI [the highest magnification of the yellow squared area shown in (A)]. There is LBC-positive intestinal metaplasia around the lesion, and the line of discontinued LBC is consistent with the border of the tumor (yellow arrows). The tumor was histologically low-grade tubular adenoma. LBC, light blue crest; NBI, narrow-band imaging.
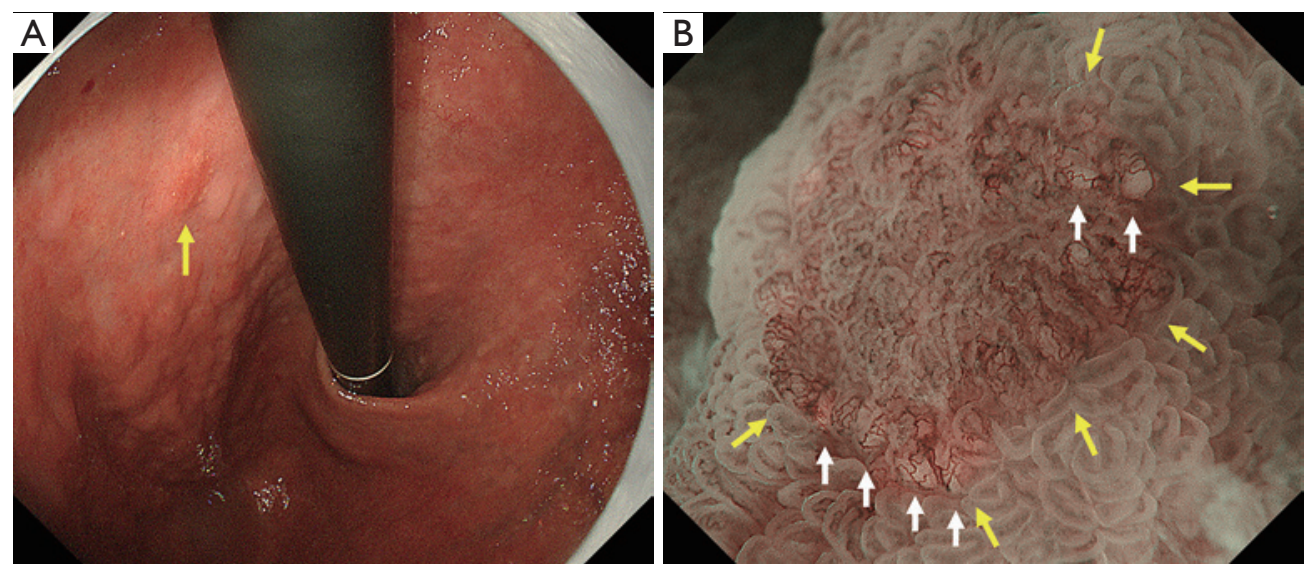

Figure 13 Case presentation of WGA. (A) Conventional endoscopic image (white light). A reddened, regional, flat mucosal lesion is present in the posterior wall of the lesser curvature in the upper part of the gastric corpus (yellow arrow), and white spots are seen in marginal areas of the lesion. (B) Magnifying endoscopic image with NBI. It was confirmed that the white spots observed on conventional endoscopy (white light) are WGAs (white arrows). WGA is characteristically found in the cancerous mucosa very close to the demarcation line (yellow arrows). WGA, white globe appearance; NBI, narrow-band imaging.

differentiated-type cancerous and non-cancerous lesions, such as low-grade adenoma and gastritis, with high specificity (Figure 13).

\section{Vessels within epithelial circle (VEC) pattern}

It has been reported that pathological examination shows higher biological malignancy of papillary adenocarcinoma than of tubular adenocarcinoma. However, using conventional endoscopy, it was not possible to diagnose papillary adenocarcinoma. Kanemitsu et al. observed early gastric cancer by magnifying endoscopy and noticed the specific finding that blood vessels were present under the superficial epithelium of intervening part surrounded by circular MCE. They named this the "vessels within epithelial circle (VEC) pattern" and reported that this pattern is characteristic 

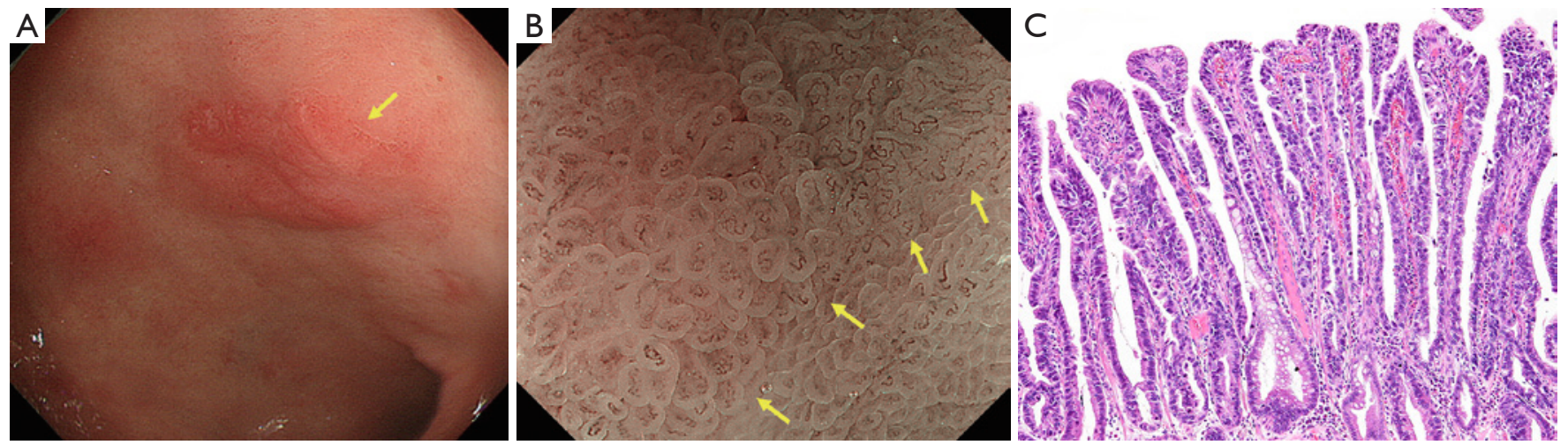

Figure 14 Case presentation of VEC pattern. (A) Conventional white light endoscopic findings of early gastric cancer (0-IIa type). A superficial elevated lesion (yellow arrow) is present on the lesser curvature of the gastric antrum. (B) Magnifying endoscopic image with NBI. Within the demarcation line (yellow arrows), the VEC pattern can be seen. An irregular microvascular pattern is observed in the circular intervening part between crypts lined by circular marginal crypt epithelium. (C) Histopathological findings. Hematoxylin and eosin (H\&E) stain (magnification $\times 40)$. Well-differentiated exophytic carcinoma with elongated finger-like processes lined by cylindrical or cuboidal cells supported by a fibrovascular connective tissue core. NBI, narrow-band imaging; VEC, vessels within epithelial circle. [Reprinted from (30)].

of a differentiated-type cancer showing histologically a papillary structure (30) (Figure 14). In addition, they reported the coexistence of undifferentiated-type cancer and/ or submucosal invasion in about one-quarter of resected histological specimens of VEC pattern-positive early gastric cancer and suggested the possibility that the VEC pattern observed on magnifying endoscopy could be a useful marker for predicting high malignancy prior to surgery (30).

In summary, a diagnostic system for image-enhanced magnifying endoscopy for early gastric cancer and other representative findings have been described in this paper. Although details have not been mentioned here, it should be emphasized that a standard technique to observe the gastric mucosa at maximum magnification is indispensable for accurate diagnosis using magnifying endoscopy.

\section{Acknowledgments}

None.

\section{Footnote}

Conflicts of Interest: The authors have no conflicts of interest to declare.

Ethical Statement: The authors are accountable for all aspects of the work in ensuring that questions related to the accuracy or integrity of any part of the work are appropriately investigated and resolved.

\section{References}

1. Yao K, Anagnostopoulos GK, Ragunath K. Magnifying endoscopy for diagnosing and delineating early gastric cancer. Endoscopy 2009;41:462-7.

2. Yao K, Iwashita A, Tanabe H, et al. White opaque substance within superficial elevated gastric neoplasia as visualized by magnification endoscopy with narrowband imaging: a new optical sign for differentiating between adenoma and carcinoma. Gastrointest Endosc 2008;68:574-80.

3. Muto M, Yao K, Kaise M, et al. Magnifying endoscopy simple diagnostic algorithm for early gastric cancer (MESDA-G). Dig Endosc 2016;28:379-93.

4. Ezoe Y, Muto M, Uedo N, et al. Magnifying narrow band imaging is more accurate than conventional whitelight imaging in diagnosis of gastric mucosal cancer. Gastroenterology 2011;141:2017-25.e3.

5. Yao K, Doyama H, Gotoda T, et al. Diagnostic performance and limitations of magnifying narrow-band imaging in screening endoscopy of early gastric cancer: a prospective multicenter feasibility study. Gastric Cancer 2014;17:669-79.

6. Yamada S, Doyama H, Yao K, et al. An efficient diagnostic 
strategy for small, depressed early gastric cancer with magnifying narrow-band imaging:a post-hoc analysis of a prospective randomized controlled trial. Gastrointest Endosc 2014;79:55-63.

7. Maki S, Yao K, Nagahama T, et al. Magnifying endoscopy with narrow-band imaging is useful in the differential diagnosis between low-grade adenoma and early cancer of superficial elevated gastric lesions. Gastric Cancer 2013;16:140-6.

8. Miwa K, Doyama H, Ito R, et al. Can magnifying endoscopy with narrow band imaging be useful for low grade adenomas in preoperative biopsy specimens? Gastric Cancer 2012;15:170-8.

9. Fujiwara S, Yao K, Nagahama T, et al. Can we accurately diagnose minute gastric cancers $(\leq 5 \mathrm{~mm})$ ? Chromoendoscopy (CE) vs magnifying endoscopy with narrow band imaging (M-NBI). Gastric Cancer 2015;18:590-6.

10. Zhang Q, Wang F, Chen ZY, et al. Comparison of the diagnostic efficacy of white light endoscopy and magnifying endoscopy with narrow band imaging for early gastric cancer: a meta-analysis. Gastric Cancer 2016;19:543-52.

11. Dohi O, Yagi N, Majima A, et al. Diagnostic ability of magnifying endoscopy with blue laser imaging for early gastric cancer: a prospective study. Gastric Cancer 2017;20:297-303.

12. Dohi O, Yagi N, Yoshida S, et al. Magnifying Blue Laser Imaging versus Magnifying Narrow-Band Imaging for the Diagnosis of Early Gastric Cancer: A Prospective, Multicenter, Comparative Study. Digestion 2017;96:127-34.

13. Yoshimizu S, Yamamoto Y, Horiuchi Y, et al. Diagnostic performance of routine esophagogastroduodenoscopy using magnifying endoscope with narrow-band imaging for gastric cancer. Dig Endosc 2018;30:71-8.

14. Yao K. Zoom gastroscopy: magnifying endoscopy in the stomach. Tokyo: Springer; 2013:66-7.

15. Yao K. The endoscopic diagnosis of early gastric cancer. Ann Gastroenterol 2013;26:11-22.

16. Matsushita M, Mori S, Uchida K, et al. "White opaque substance" and "light blue crest" within gastric flat tumors or intestinal metaplasia: same or different signs? Gastrointest Endosc 2009;70:402-3.

17. Kanemitsu T, Yao K, Nagahama T, et al. Extending magnifying NBI diagnosis of intestinal metaplasia in the stomach: the white opaque substance marker. Endoscopy
2017;49:529-35.

18. Yao K, Iwashita A, Nambu M, et al. Nature of white opaque substance in gastric epithelial neoplasia as visualized by magnifying endoscopy with narrow-band imaging. Dig Endosc 2012;24:419-25.

19. Ueo T, Yonemasu H, Yada N, et al. White opaque substance represents an intracytoplasmic accumulation of lipid droplets: immunohistochemical and immunoelectron microscopic investigation of 26 cases. Dig Endosc 2013;25:147-55.

20. Ueo T, Yonemasu H, Yao K, et al. Histologic differentiation and mucin phenotype in white opaque substance-positive gastric neoplasias. Endosc Int Open 2015;3:E597-604.

21. Song J, Zhang J, Wang J, et al. Meta-analysis: narrow band imaging for diagnosis of gastric intestinal metaplasia. PLoS One 2014;9:e94869.

22. Ang TL, Fock KM, Teo EK, et al. The diagnostic utility of narrow band imaging magnifying endoscopy in clinical practice in a population with intermediate gastric cancer risk. Eur J Gastroenterol Hepatol 2012;24:362-7.

23. Lage J, Pimentel-Nunes P, Figueiredo PC, et al. LightNBI to identify high-risk phenotypes for gastric adenocarcinoma: do we still need biopsies? Scand J Gastroenterol 2016;51:501-6.

24. Dutta AK, Sajith KG, Pulimood AB, et al. Narrow band imaging versus white light gastroscopy in detecting potentially premalignant gastric lesions: a randomized prospective crossover study. Indian J Gastroenterol 2013;32:37-42.

25. Uedo N, Ishihara R, Iishi $H$, et al. A new method of diagnosing gastric intestinal metaplasia: narrowband imaging with magnifying endoscopy. Endoscopy 2006;38:819-824.

26. Savarino E, Corbo M, Dulbecco P, et al. Narrowband imaging with magnifying endoscopy is accurate for detecting gastric intestinal metaplasia. World J Gastroenterol 2013;19:2668-75.

27. Rerknimitr R, Imraporn B, Klaikeaw N, et al. Nonsequential narrow band imaging for targeted biopsy and monitoring of gastric intestinal metaplasia. World J Gastroenterol 2011;17:1336-42.

28. Wang L, Huang W, Du J, et al. Diagnostic yield of the light blue crest sign in gastric intestinal metaplasia: a metaanalysis. PLoS One 2014;9:e92874.

29. Doyama H, Yoshida N, Tsuyama S, et al. The "white globe appearance" (WGA): a novel marker for a correct 
diagnosis of early gastric cancer by magnifying endoscopy with narrow-band imaging (M-NBI). Endosc Int Open 2015;3:E120-4.

30. Kanemitsu T, Yao K, Nagahama T, et al. The vessels within epithelial circle (VEC) pattern as visualized by

doi: $10.21037 / \operatorname{tgh} .2019 .12 .16$

Cite this article as: Miyaoka M, Yao K, Tanabe H, Kanemitsu T, Otsu K, Imamura K, Ono Y, Ishikawa S, Yasaka T, Ueki T, Ota A, Haraoka S, Iwashita A. Diagnosis of early gastric cancer using image enhanced endoscopy: a systematic approach. Transl Gastroenterol Hepatol 2020;5:50. magnifying endoscopy with narrow-band imaging (ME$\mathrm{NBI}$ ) is a useful marker for the diagnosis of papillary adenocarcinoma: a case-controlled study. Gastric Cancer 2014;17:469-77. 